\title{
Lattice Boltzmann simulation of liquid-gas flows through solid bodies in a square duct
}

\author{
Masato Yoshino ${ }^{*}$, Yusuke Mizutani ${ }^{1}$ \\ Department of Mechanical Systems Engineering, Faculty of Engineering, \\ Shinshu University, 4-17-1 Wakasato, Nagano 380-8553, Japan
}

\begin{abstract}
The lattice Boltzmann method for two-phase immiscible fluids with large density differences proposed by Inamuro et al. [J. Comput. Phys. 198 (2004) 628-644] is applied to the problem of liquid-gas flows through solid bodies in a square duct. A wetting boundary condition is introduced so that partial wetting on solid surfaces is realized to agree with Cahn theory. Using this method, we investigate the characteristics of wettability in terms of dynamic contact angles between two fluids and a solid wall. Also, we carry out simulations of liquid-gas rising flows through solid bodies in a square duct. It is found from these simulations that the present method can be useful for the problems of liquid-gas flows through complicated geometries.
\end{abstract}

Key words: Lattice Boltzmann method (LBM), Bubble flows, Wetting dynamics, Contact angles

\section{Introduction}

The problems of liquid-gas two-phase flows are of great importance in many engineering and industrial fields, such as boiling systems, nuclear reactors, and chemical plants. In the past studies on bubble flows, for example, Grace [1] and Bhaga and Weber [2] made experimental investigation into shapes and terminal velocities of bubbles rising in viscous liquids. With the aid of remarkable progress in computers, numerical simulations are currently used to understand fluid dynamics in two-phase fluid flows which cannot be readily examined by experimental approaches. In particular, the lattice Boltzmann method (LBM) has been developed into an alternative and promising numerical scheme for simulating multiphase fluid flows. Swift et al. [3] developed an LBM model for multiphase fluid flows using the free-energy approach. Inamuro et al. [4]

* Corresponding author. Tel: +81-26-269-5116; fax: +81-26-269-5116.

Email address: masato@shinshu-u.ac.jp (Masato Yoshino).

1 Present address: NIDEC Sankyo Corp., Simo-Suwa, Nagano 393-8511, Japan. 
proposed an LBM for incompressible two-phase flows with large density differences. It is reported in [5] that one can simulate two-phase fluid flows with the density ratio up to 1000 using this method. On the other hand, two-phase flows through solid bodies, such as porous media, are also interesting subjects. Although the above-mentioned methods can be applied to such problems, it is needed to take account of wettability on solid surfaces and to incorporate an appropriate condition into the lattice Boltzmann scheme.

In this paper, the LBM for two-phase immiscible fluids with large density differences [4], together with the wetting boundary condition proposed by Briant et al. [6,7], is applied to the simulations of liquid-gas flows through solid bodies in a square duct. The bubble behavior and velocity fields around solid bodies are simulated under different wetting conditions.

\section{Numerical Method and Conditions}

\subsection{Two-phase lattice Boltzmann method}

Non-dimensional variables, defined by using a characteristic length $L$, a characteristic particle speed $c$, a characteristic time scale $t_{0}=L / U$ where $U$ is a characteristic flow speed, a reference order parameter $\phi_{0}$, and a reference density $\rho_{0}$, are used as in [4]. The fifteen-velocity model is used in the computations. The algorithm of computation is described in detail in [4].

\subsection{Wetting boundary condition}

Recently, Briant et al. $[6,7]$ have proposed a wetting boundary condition which enables the contact angle of the interface to be controlled in a way consistent with Cahn theory [8]. In their method, the derivative of the density normal to the wall, $\partial \rho / \partial n$, is specified using the wetting potential which is calculated according to a desired static contact angle, $\theta_{\mathrm{w}}$. In the following computations, the specified value of $\partial \rho / \partial n$ at wall sites is substituted for the first and second derivatives in the Chapman-Enskog type functions, $f_{i}^{\mathrm{c}}$ and $g_{i}^{\mathrm{c}}$, defined in [4].

To confirm the validity of the method, we conducted preliminary calculations of a bubble on a flat plate with different wettability. Figure 1 shows the comparison of calculated static contact angles with theoretical values by Young [9]. It is found that the present results are in good agreement with the theoretical ones for $70^{\circ} \leq \theta_{\mathrm{w}} \leq 110^{\circ}$.

\subsection{Computational domain and conditions}

We apply the method to problems of a rising bubble past a solid body and of a rising bubble through multibodies. In both problems, a rectangular domain whose size is $L_{x}=L_{y}=0.5 L_{z}$ is considered. The domain is filled with liquid from bottom up to $0.94 L_{z}$ height, and the rest of the domain is regarded as gas 
phase. The gravitational force is assumed to act in the negative $z$-direction. In the first problem, a thin body of $0.050 L_{x} \times 0.85 L_{y} \times 0.38 L_{z}$ is considered and the center is positioned at $\left(x / L_{x}, y / L_{y}, z / L_{z}\right)=(0.50,0.50,0.59)$ in the domain. Then, a single bubble with diameter $D=0.3 L_{z}$ is placed at $\left(x / L_{x}, y / L_{y}, z / L_{z}\right)=(0.50,0.50,0.20)$ and is released at time $t=0$. In the second problem, ten identical cubic bodies with sides of $0.075 L_{z}$ are arbitrarily arranged in the square duct. The centers of the bodies are $\left(x / L_{x}, y / L_{y}, z / L_{z}\right)$ $=(0.25,0.25,0.38),(0.25,0.75,0.38),(0.75,0.25,0.38),(0.75,0.75,0.38)$, $(0.40,0.50,0.49),(0.50,0.73,0.55),(0.60,0.35,0.64),(0.23,0.55,0.70),(0.85$, $0.63,0.63)$, and $(0.35,0.23,0.71)$. A single bubble with diameter $D=0.35 L_{z}$ is placed at $\left(x / L_{x}, y / L_{y}, z / L_{z}\right)=(0.50,0.50,0.21)$ and is released at time $t=0$. The periodic boundary condition is used on the sides of the domain. The bounce-back boundary condition is used at the top and bottom walls and on the surface of the bodies. In addition, the wetting condition in Sec. 2.2 is imposed on the surface of the bodies.

The conditions in the present simulations are as follows. The whole domain is divided into a $40 \times 40 \times 80$ cubic lattice. The parameters determining the maximum and minimum values of the order parameter are $a=1, b=1$, and $T=2.93 \times 10^{-1}$; it follows that these values are $\phi_{\max }=4.031 \times 10^{-1}$ and $\phi_{\text {min }}=2.638 \times 10^{-1}$, respectively. The cut-off values of the order parameter for obtaining the density in the interface are $\phi_{\mathrm{L}}^{*}=3.80 \times 10^{-1}$ and $\phi_{\mathrm{G}}^{*}=2.75 \times$ $10^{-1}$. The other parameters are fixed at $\tau_{f}=1, \tau_{g}=1, \varepsilon=10^{-5}, \rho_{\mathrm{L}}=50$, $\rho_{\mathrm{G}}=1\left(\rho_{\mathrm{L}} / \rho_{\mathrm{G}}=50\right), \mu_{\mathrm{L}}=1 \times 10^{-2} \Delta x, \mu_{\mathrm{G}}=2 \times 10^{-4} \Delta x, \kappa_{f}=0.5(\Delta x)^{2}$, and $\kappa_{g}=1 \times 10^{-5}(\Delta x)^{2}$, where $\tau_{f}$ and $\tau_{g}$ are dimensionless single relaxation times, $\varepsilon$ is the convergence criterion for the pressure correction, $\Delta x$ is a spacing of the cubic lattice, $\rho_{\mathrm{L}}$ and $\rho_{\mathrm{G}}$ are the densities of liquid and gas, $\mu_{\mathrm{L}}$ and $\mu_{\mathrm{G}}$ are the viscosities of liquid and gas, and $\kappa_{f}$ and $\kappa_{g}$ are constants determining the width of the interface and the strength of the surface tension, respectively. As in [10], the dimensionless parameters for these phenomena are the Bond number $\mathrm{B}=\rho_{\mathrm{L}} g D^{2} / \sigma$, the capillary number $\mathrm{Ca}=\mu_{\mathrm{L}} V_{0} / \sigma$, and the Reynolds number $\operatorname{Re}=\rho_{\mathrm{L}} V_{0} D / \mu_{\mathrm{L}}$, where $g$ is the gravitational acceleration, $\sigma$ is the surface tension, and $V_{0}$ is a superficial velocity of gas phase in the steady state.

\section{Results and Discussion}

\subsection{A rising bubble past a solid body}

First, in order to investigate dynamic contact angles, we calculate behavior of a rising bubble past a solid body. We carry out the simulations for three static contact angles $\left(\theta_{\mathrm{w}}=70^{\circ}, 90^{\circ}\right.$, and $\left.110^{\circ}\right)$. Hereafter, let $\theta_{\mathrm{a}}$ and $\theta_{\mathrm{r}}$ be advancing and receding dynamic contact angles, respectively. Figure 2 shows the time variation of $\theta_{\mathrm{a}}$ and $\theta_{\mathrm{r}}$ while the whole bubble is in contact with the body. In all cases, $\theta_{\mathrm{a}}$ remains almost constant as time goes on. On the other hand, $\theta_{\mathrm{r}}$ decreases in the early stages and then approaches a certain value. Moreover, it 


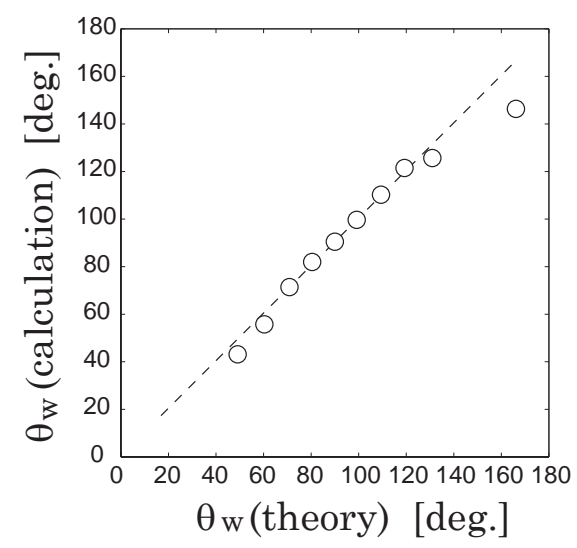

Fig. 1. Comparison of calculated static contact angles with theoretical values by Young [9]. The dashed line represents an incline of 1 .

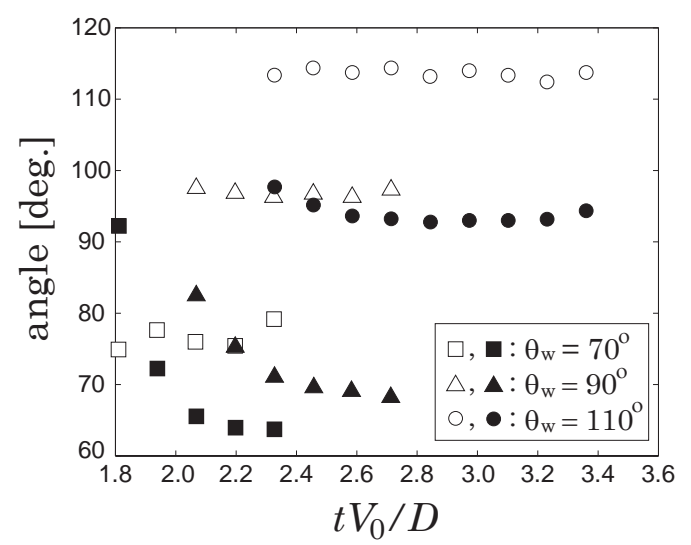

Fig. 2. Dynamic contact angles of riseing bubble past solid body. The open and closed symbols indicate $\theta_{\mathrm{a}}$ and $\theta_{\mathrm{r}}$, respectively.

is seen that the rate of decrease is larger as $\theta_{\mathrm{w}}$ becomes lower. The calculated terminal contact angles become $\left(\theta_{\mathrm{a}}, \theta_{\mathrm{r}}\right)=\left(79.2^{\circ}, 63.8^{\circ}\right),\left(97.3^{\circ}, 68.8^{\circ}\right)$, and $\left(113.8^{\circ}, 94.4^{\circ}\right)$ for $\theta_{\mathrm{w}}=70^{\circ}, 90^{\circ}$, and $110^{\circ}$, respectively. It is found that the relation of $\theta_{\mathrm{r}}<\theta_{\mathrm{w}}<\theta_{\mathrm{a}}$ is obtained and that the tendency of time variation in dynamic contact angles is similar to that in experimental results by Sikalo et al. [11] .

Figure 3 shows the calculated results of bubble behavior and velocity fields on $y=0.5 L_{y}$ for $\theta_{\mathrm{w}}=70^{\circ}$ and $110^{\circ}$. Note that the bubble is apart from the body in the initial state. In both cases, it is seen that after the collision with the body, the bubble slides on the solid surface and then goes up to the free surface at the top. Comparing with these cases, we can see that the shapes of the bubble are different. In the case of $\theta_{\mathrm{w}}=70^{\circ}$, the bubble spreads along the body until it reaches the top surface. In the case of $\theta_{\mathrm{w}}=110^{\circ}$, on the other hand, the bubble tends to keep the spherical shape in spite of slight deformation.

\subsection{A rising bubble through multibodies}

Next, we perform simulations of a rising bubble through multibodies in a square duct. Figure 4 shows the calculated results of bubble behavior and velocity fields on $y=0.37 L_{y}$ for $\theta_{\mathrm{w}}=70^{\circ}$ and $110^{\circ}$. It is seen that the bubble is deformed by the bodies and the velocity fields around the bubble are very complicated in both cases. However, as for the path of the rising bubble, there are differences between these cases. In the case of $\theta_{\mathrm{w}}=70^{\circ}$, the bubble rises in the relatively central region of the duct, while in the case of $\theta_{\mathrm{w}}=110^{\circ}$, the bubble avoids the bodies and goes up through open spaces. Thus, it is found that differences in wettability on solid surface give quite different bubble behavior and flow fields in such a problem. 

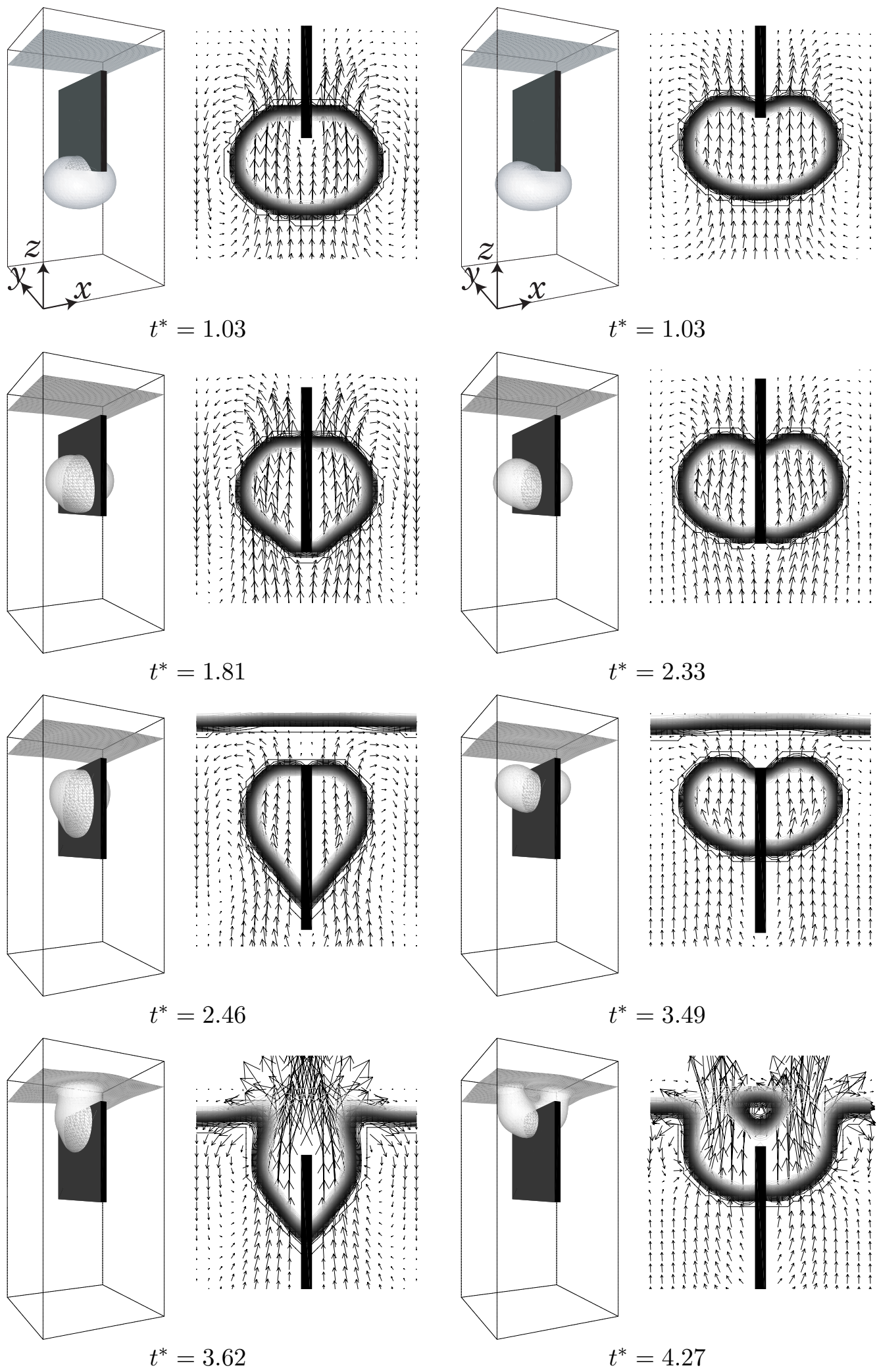

(a) $\theta_{\mathrm{w}}=70^{\circ}$

(b) $\theta_{\mathrm{w}}=110^{\circ}$

Fig. 3. Time evolution of bubble shape (left) and velocity vectors and density contours on $y=0.5 L_{y}$ (right) for (a) $\theta_{\mathrm{w}}=70^{\circ}$, (b) $\theta_{\mathrm{w}}=110^{\circ}$. The dimensionless numbers are $\mathrm{B}=3.60, \mathrm{Ca}=3.88 \times 10^{-3}$, and $\mathrm{Re}=372\left(t^{*}=t V_{0} / D\right.$, where $V_{0}$ is the superficial velocity). 

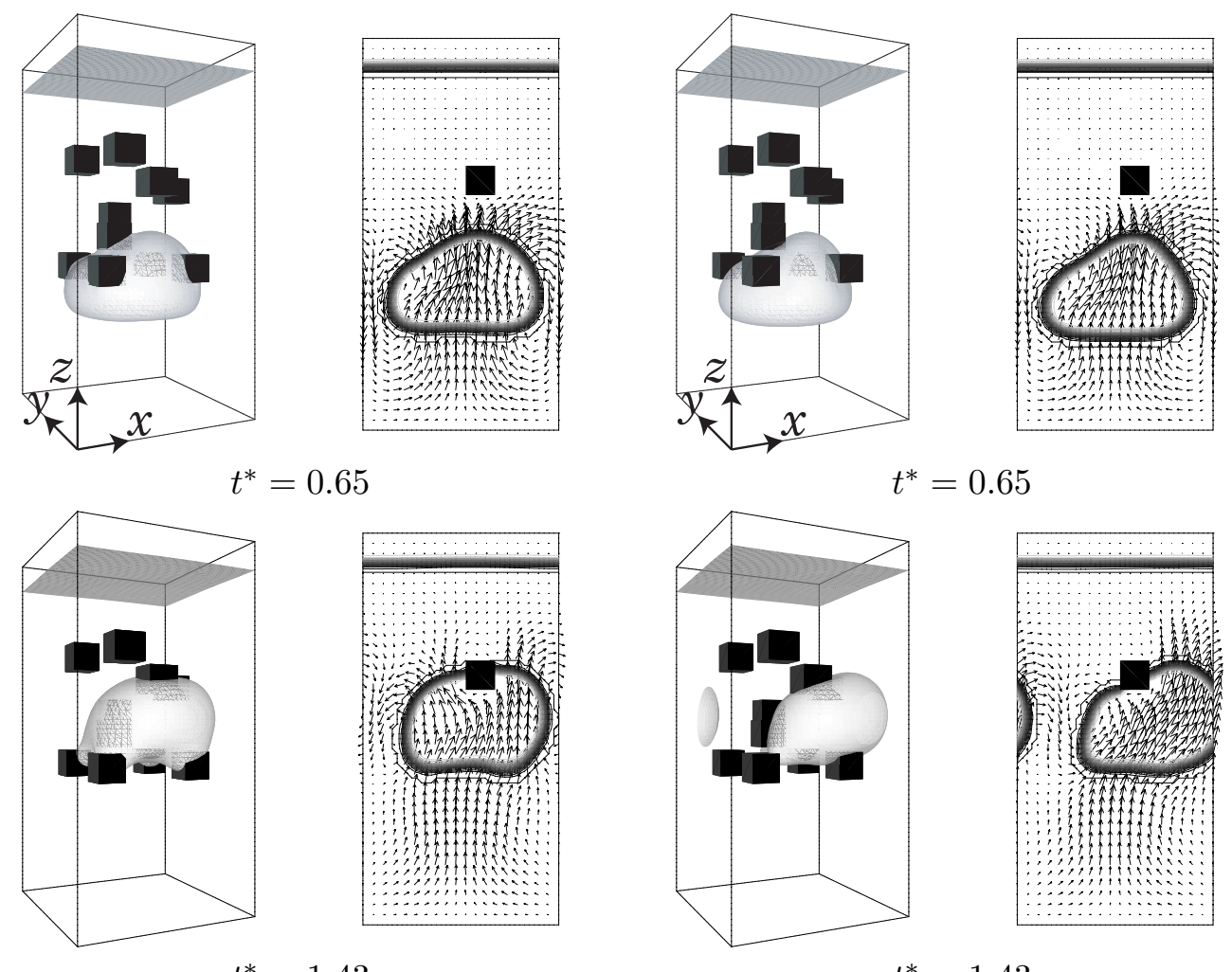

$t^{*}=1.43$

$$
t^{*}=1.43
$$
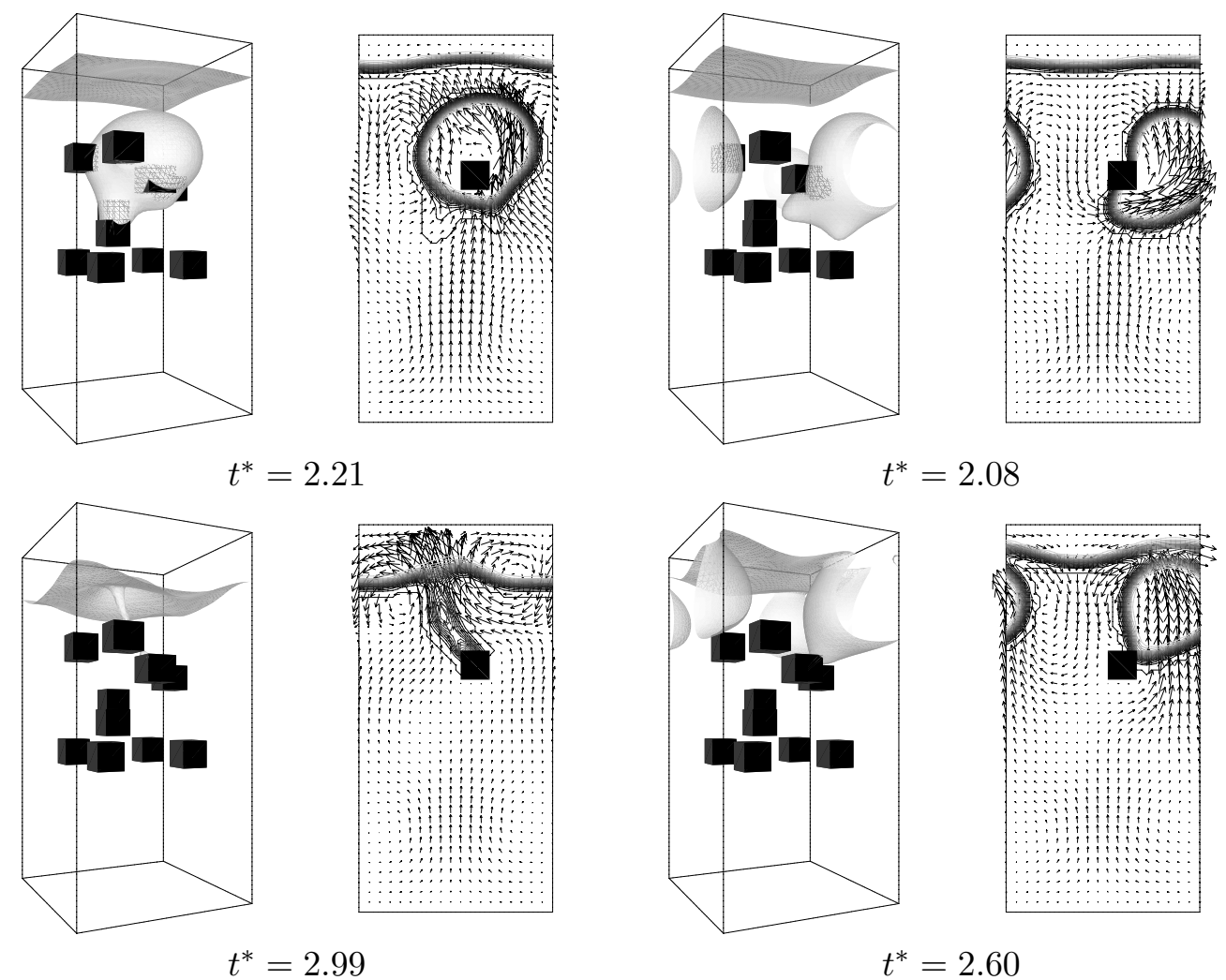

(a) $\theta_{\mathrm{w}}=70^{\circ}$

(b) $\theta_{\mathrm{w}}=110^{\circ}$

Fig. 4. Time evolution of bubble shape (left) and velocity vectors and density contours on $y=0.37 L_{y}$ (right) for (a) $\theta_{\mathrm{w}}=70^{\circ}$, (b) $\theta_{\mathrm{w}}=110^{\circ}$. The dimensionless numbers are $\mathrm{B}=9.80, \mathrm{Ca}=4.55 \times 10^{-3}$, and $\mathrm{Re}=510\left(t^{*}=t V_{0} / D\right.$, where $V_{0}$ is the superficial velocity). 


\section{Concluding Remarks}

The LBM for two-phase immiscible fluids with large density differences, together with the wetting boundary condition, is applied to simulations of liquidgas flows in a square duct including solid bodies. The bubble behavior and complicated velocity fields around the bodies are obtained for different wettabilities on the solid surface. From these results, it is found that the present method can be a useful tool for simulating liquid-gas flows through complicated geometries. Finally, the effect of dimensionless numbers such as the Bond number on bubble behavior and velocity fields was not carefully examined in the present work. Also, the porosity of porous structures would depend on these phenomena. The studies of such investigations are required in the future.

\section{Acknowledgments}

This research was partly supported by the Grant-in-Aid (No. 16760122) for Scientific Research from the Ministry of Education, Culture, Sports, Science, and Technology in Japan. The authors thank the anonymous referees for helpful suggestions on the manuscript.

\section{References}

[1] J.R. Grace, T. Wairegi, T.H. Nguyen, Shapes and velocities of single drops and bubbles moving freely through immiscible liquids, Trans. Inst. Chem. Eng. 54 (1976) 167-173.

[2] D. Bhaga, M.E. Weber, Bubbles in viscous liquids: shapes, wakes and velocities, J. Fluid Mech. 105 (1981) 61-85.

[3] M.R. Swift, W.R. Osborn, J.M. Yeomans, Lattice Boltzmann simulation of nonideal fluids, Phys. Rev. Lett. 75 (1995) 830-833.

[4] T. Inamuro, T. Ogata, S. Tajima, N. Konishi, A lattice Boltzmann method for incompressible twophase flows with large density differences, J. Comput. Phys. 198 (2004) 628-644.

[5] T. Inamuro, T. Ogata, F. Ogino, Numerical simulation of bubble flows by the lattice Boltzmann method, Future Gener. Comput. Syst. 20 (2004) 959-964.

[6] A.J. Briant, P. Papatzacos, J.M. Yeomans, Lattice Boltzmann simulations of contact line motion in a liquid-gas system, Philos. Trans. R. Soc. Lond. Ser. A 360 (2002) 485-495.

[7] A.J. Briant, A.J. Wagner, J.M. Yeomans, Lattice Boltzmann simulations of contact line motion I. Liquid-gas systems, Phys. Rev. E 69 (2004) 031602.

[8] J.W. Cahn, Critical point wetting, J. Chem. Phys. 66 (1977) 3667-3672.

[9] T. Young, An essay on the cohesion of fluids, Philos. Trans. R. Soc. Lond. Ser. A 95 (1805) 65-87.

[10] C.E. Norman, M.J. Miksis, Gas bubble with a moving contact line rising in an inclined channel at finite Reynolds number, Physica D 209 (2005) 191-204.

[11] Š. Šikalo, C. Tropea, E.N. Ganić, Dynamic wetting angle of a spreading droplet, Exp. Therm. Fluid Sci. 29 (2005) 795-802. 